\title{
GnRH Agonist Ovulation Trigger in Patients Undergoing Controlled Ovarian Hyperstimulation for IVF with Stop GnRH-agonist Combined With Multidose GnRH-antagonist Protocol.
}

Raoul Orvieto ( $\square$ raoul.orvieto@sheba.health.gov.il )

Sheba Medical Center at Tel Hashomer

Ravit Nahum

Sheba Medical Center at Tel Hashomer

Judith Frei

Sheba Medical Center at Tel Hashomer

Orit Zandman

Sheba Medical Center at Tel Hashomer

Yulia Frenkel

Sheba Medical Center at Tel Hashomer

Jigal Haas

TRIO fertility partners https://orcid.org/0000-0001-5274-7358

\section{Research}

Keywords: Stop GnRH-agonist, Multidose $\mathrm{GnRH}$-antagonist, $\mathrm{COH}, \mathrm{GnRH}$-agonist, Ovulation trigger,

Suboptimal response, LH

Posted Date: January 22nd, 2021

DOl: https://doi.org/10.21203/rs.3.rs-151943/v1

License: (c) (i) This work is licensed under a Creative Commons Attribution 4.0 International License.

Read Full License

Version of Record: A version of this preprint was published at Gynecologic and Obstetric Investigation on September 17th, 2021. See the published version at https://doi.org/10.1159/000517177. 


\section{Abstract}

Backround: Recently, the Stop GnRH agonist protocol has been used successfully in poor responder patients, those with poor embryos quality and those with elevated peak serum progesterone levels. The aim of the present study was to evaluate, whether GnRH-agonist trigger in patients undergoing the Stop protocol combined, will result in an optimal response/trigger, as reflected by post trigger $\mathrm{LH}>15 \mathrm{mlU} / \mathrm{mL}$

Methods: A retrospective cohort study. All consecutive women admitted to our IVF unit from February 2020 through November 2020 who reached the ovum pick-up stage. Patients triggered with GnRH-ag alone, or combined with hCG for final oocyte maturation were included in the study. LH levels were measured 12 hours post trigger.

Results: Five out of the 32 patients (15.6\%) demonstrated suboptimal response as reflected by LH levels $<15 \mathrm{IU} / \mathrm{L} 12 \mathrm{hrs}$ post GnRH-agonist trigger. Moreover, while no differences were observed in oocytes recovery rate, maturity or embryos quality between the different study groups, those achieving a suboptimal response to the $\mathrm{GnRH}$-agonist trigger (post trigger $\mathrm{LH}<15 \mathrm{mlU} / \mathrm{mL}$ ) demonstrated significantly higher number of follicles and peak estradiol levels at the day of trigger.

Conclusions: The Stop GnRH-agonist combined with GnRH-antagonist protocol, enables the substitution of HCG with GnRH-ag for final oocyte maturation. However, caution should be taken in high responders, where the dual trigger with small doses of hCG (1000-1500IU) should be considered, aiming to avoid suboptimal response (post trigger LH levels $<15 \mathrm{IU} / \mathrm{L}$ ).

\section{Background}

Controlled ovarian hyperstimulation $(\mathrm{COH})$ enables the recruitment of multiple healthy fertilizable oocytes in patients undergoing in vitro fertilization-embryo transfer (IVF-ET) (1). However, despite the great improvements in ovarian stimulation protocols and fertilization procedures, live birth rates per embryo transferred remain at approximately $15-50 \%$ and severe ovarian hyperstimulation syndrome (OHSS) remains one of their major threats $(2-3)$.

Moreover, while there are no precise methods to completely prevent severe OHSS, except by withholding human chorionic gonadotropin ( $\mathrm{hCG}$ ), during the last decade, observations have suggested that gonadotropin-releasing hormone agonist ( $\mathrm{GnRH}-\mathrm{ag}$ ) by its consequent $\mathrm{LH}$ surge, may effectively trigger oocyte maturation and ovulation, with the consequent elimination of severe OHSS (4-5). It is noteworthy, that previous studies have shown that patients receiving a $\mathrm{GnRH}$-agonist trigger alone, who have a post trigger $\mathrm{LH}<15 \mathrm{mIU} / \mathrm{mL}$, were more likely to have suboptimal response or a cancelled retrieval (6-9).

Recently, we introduced the Stop GnRH-Agonist combined with multiple-dose $\mathrm{GnRH}$-antagonist protocol to our $\mathrm{COH}$ armamentarium. The rationale behind the sequential treatment stems from the advantages of its components. The mid-luteal $\mathrm{GnRH}$-ag pre-treatment causes down regulation of the $\mathrm{GnRH}$ receptors with the consequent suppression of pituitary LH secretion for as long as 10 days after the last dose of the 
agonist. This effect, together with the immediate LH suppression provided by the $\mathrm{GnRH}$-ant, will eliminate premature LH surge and might improve the quality of the embryos generated (10).

This protocol was successfully used in poor responder patients (10), those with poor embryos quality (11) and those with elevated peak serum progesterone levels (12). Moreover, following the observations of comparable or improved oocyte/embryos quality following GnRH-a trigger as compared to hCG, and the different effects of $\mathrm{LH}$ and $\mathrm{hCG}$ on the downstream signaling of the $\mathrm{LH}$ receptor, $\mathrm{GnRH}-\mathrm{a}$ is now offered concomitantly with the standard hCG trigger dose (dual trigger), to improve oocyte/embryo yield and quality (13), or solely, instead of HCG, aiming to eliminate the risk to develop severe OHSS (3).

The aim of the present study was to evaluate, whether $\mathrm{GnRH}$-agonist trigger in patients undergoing the Stop GnRH-Agonist combined with multiple-dose $\mathrm{GnRH}$-antagonist protocol, will result in an optimal response/trigger, as reflected by post trigger $\mathrm{LH}<15 \mathrm{mIU} / \mathrm{mL}(6-9)$.

\section{Patients And Methods}

We reviewed the computerized files of all consecutive women admitted to our IVF unit from February 2020 through November 2020 who reached the ovum pick-up stage. Exclusion criteria were use of donor oocytes or transfer of frozen-thawed embryos, and use of other than the Stop GnRH-Agonist combined with multiple-dose $\mathrm{GnRH}$-antagonist protocol [as previously described (10)]. Of whom, only those patients who underwent triggering of final follicular maturation with $\mathrm{GnRH}$-agonist trigger, either solely or combined with hCG (dual trigger), were included. LH levels were measured 12 hours post GnRH-agonist trigger injection. Those with post trigger $\mathrm{LH}<15 \mathrm{mIU} / \mathrm{mL}$ were considered as achieving a suboptimal response. The study was approved by the institutional research ethics board of Sheba Medical Center.

Data on patient age and infertility-treatment-related variables were collected from the files. The decision regarding final follicular maturation triggering was based on physician judgement. Generally, patients exhibiting Estradiol level $>10,000 \mathrm{pmol} / \mathrm{L}$ were prescribed the $\mathrm{GnRH}$-agonist only trigger, as were patients developing $>15$ follicles of $>10 \mathrm{~mm}$ diameter. LH level 12 hours post trigger, embryological parameters and number of oocyte retrieved and oocyte recovery rate (defined as the number of oocyte retrieved per number of follicles $>10 \mathrm{~mm}$ in diameter at the day of trigger) were also retrieved and analyzed. Classification of embryo quality was based on previously published scoring parameters (14); a topquality embryo was defined as four to five blastomeres on day 2 , seven or more blastomeres on day 3 , equally-sized blastomeres and $\leq 10 \%$ fragmentation on day 3 and no multinucleation.

Statistical analysis was performed with Student's t-test and Chi square, as appropriate. Results are presented as means \pm standard deviations; $p$ value $<0.05$ was considered significant.

\section{Results}

Of the 1070 cycles performed in our IVF unit from February 2020 through November 2020, 32 IVF cycles were eligible for analysis. Patients' age, BMI and basal Day-3 FSH levels were $39.3 \pm 3.9$ yrs, $22.7 \pm 4.2$ 
$\mathrm{kg} / \mathrm{M}^{2}$, and $11.0 \pm 4.7 \mathrm{IU} / \mathrm{L}$, respectively. Five patients underwent the $\mathrm{GnRH}$-agonist trigger solely, and 27 the dual trigger, of whom 1 and 4 presented a suboptimal $\mathrm{LH}$ rise following the $\mathrm{GnRH}$-agonist trigger, respectively. The clinical characteristics of the IVF cycles of these two groups are shown in Table 1. As expected, those undergoing the $\mathrm{GnRH}$-agonist solely trigger demonstrated significantly higher peak Estradiol level $(10303 \pm 9354$ vs $4507 \pm 3924$, respectively. $P<0.02)$ and more follicles $<14 \mathrm{~mm}$ in diameter at the trigger day $(8.4 \pm 6.4$ vs $4.6 \pm 3.3$, respectively. $P<0.03)$. These differences are biased owing to the fact that we offered the $\mathrm{GnRH}$-agonist solely trigger approach mainly to patients at risk to develop OHSS or those destined to freeze-all (such as those undergoing preimplantation genetic testing). No in-between groups difference were observed in the number of oocytes retrieved, oocytes recovery rates or the percentage of MIl oocytes per oocytes retrieved, nor the percentages of 2PN per MII oocytes or TQE per 2PN. 


\begin{tabular}{|llll|}
\hline & $\begin{array}{l}\text { GnRH-agonist } \\
\text { trigger } \\
(\mathbf{n}=\mathbf{5})\end{array}$ & $\begin{array}{l}\text { Dual } \\
\text { trigger } \\
(\mathbf{n}=\mathbf{2 7})\end{array}$ & $\begin{array}{l}\mathbf{P} \\
\text { value }\end{array}$ \\
\hline Age & $38.6 \pm 3.2$ & $39.5 \pm 4.1$ & 0.32 \\
\hline Day-3 FSH (IU/L) & $8.9 \pm 2.1$ & $11.5 \pm 5.2$ & 0.21 \\
\hline BMI (kg/m ${ }^{2}$ ) & $20.5 \pm 3.3$ & $23.1 \pm 4.3$ & 0.13 \\
\hline Duration of stimulation (days) & $11.2 \pm 1.9$ & $11.2 \pm 2.3$ & 0.49 \\
\hline Total dose of FSH used (IU) & $4310 \pm 2309$ & $5068 \pm$ & 0.15 \\
\hline Peak E2 levels (pmol/L) & $10303 \pm 9354$ & $4507 \pm$ & 0.02 \\
\hline Peak Progesterone level (nmol/L) & & 3924 & \\
\hline \# follicles $>14$ mm in diameter at day of trigger & $8.4 \pm 6.4$ & $1.75 \pm 0.66$ & 0.08 \\
\hline \# follicles $>10$ mm in diameter at day of trigger & $10.2 \pm 8.4$ & $4.6 \pm 3.35$ & 0.03 \\
\hline Post trigger LH levels (IU/L) & $48 \pm 29$ & $7.3 \pm 5.2$ & 0.12 \\
\hline Interval between last GnRH-agonist and trigger day & $15.4 \pm 1.3$ & $38 \pm 20$ & 0.18 \\
\hline days) & & $13.9 \pm 2.6$ & 0.12 \\
\hline \# of oocytes retrieved & $7 \pm 5.6$ & $7.5 \pm 6.5$ & 0.4 \\
\hline Oocyte recovery rate & $0.66 \pm 0.28$ & $0.99 \pm 0.49$ & 0.14 \\
\hline \% of MII oocytes per oocytes retrieved & $0.8 \pm 0.18$ & $0.8 \pm 0.2$ & 0.48 \\
\hline \% of 2PN per MII oocytes retrieved & $0.52 \pm 0.27$ & $0.62 \pm 0.29$ & 0.29 \\
\hline \% of TQE per 2PN & $0.62 \pm 0.2$ & $0.56 \pm 0.54$ & 0.43 \\
\hline
\end{tabular}

While comparing those with post trigger $\mathrm{LH}<15 \mathrm{mlU} / \mathrm{mL}$, who were considered as achieving a suboptimal response to those with optimal response ( $\mathrm{LH}>15 \mathrm{IU} / \mathrm{L}$ ), no in-between groups differences were observed in the number of oocytes retrieved, oocytes recovery rates or the percentage of MII oocytes per oocytes retrieved, nor the percentages of 2PN per MII oocytes or TQE per 2PN (Table 2). While considering ovarian-stimulation characteristics, those achieving a suboptimal response to the $\mathrm{GnRH}$ agonist trigger demonstrated significantly higher number of follicles and peak estradiol levels (11413+ 8106 vs $4301+3601 \mathrm{pmol} / \mathrm{L}$, respectively. $\mathrm{p}<0.003$ ) at the day of trigger (Table 2 ). 


\begin{tabular}{|c|c|c|c|}
\hline & $\begin{array}{l}\text { Suboptimal } \\
\text { response } \\
(n=5)\end{array}$ & $\begin{array}{l}\text { Optimal } \\
\text { response } \\
(n=27)\end{array}$ & $\begin{array}{l}P \\
\text { value }\end{array}$ \\
\hline Age & $39.2 \pm 5.2$ & $39.3 \pm 3.5$ & 0.46 \\
\hline Day-3 FSH (IU/L) & $7.8 \pm 0.8$ & $11.5 \pm 4.8$ & 0.16 \\
\hline BMI $\left(\mathrm{kg} / \mathrm{m}^{2}\right)$ & $24.2 \pm 4.1$ & $22.5 \pm 4.2$ & 0.23 \\
\hline Duration of stimulation (days) & $11.6 \pm 1.0$ & $11.1 \pm 2.3$ & 0.32 \\
\hline Total dose of FSH used (IU) & $5000 \pm 1558$ & $4940 \pm 1445$ & 0.46 \\
\hline Peak E2 levels (pmol/L) & $11413 \pm 8106$ & $4301 \pm 3601$ & $\begin{array}{l}<.003 \\
0.03\end{array}$ \\
\hline Peak Progesterone level (nmol/L) & $1.8 \pm 0.9$ & $1.8 \pm 0.66$ & 0.47 \\
\hline \# follicles $>14 \mathrm{~mm}$ in diameter at day of trigger & $9.2 \pm 6.1$ & $4.5 \pm 2.9$ & $\begin{array}{l}< \\
0.008\end{array}$ \\
\hline \# follicles $>10 \mathrm{~mm}$ in diameter at day of trigger & $13 \pm 7.1$ & $6.7 \pm 4.7$ & $<0.02$ \\
\hline Post trigger LH levels (IU/L) & $10.4 \pm 2.1$ & $44.9 \pm 19.1$ & $\begin{array}{l}< \\
0.001\end{array}$ \\
\hline $\begin{array}{l}\text { Interval between last } \mathrm{GnRH} \text {-agonist and trigger day } \\
\text { (days) }\end{array}$ & $13.4 \pm 1.7$ & $14.3 \pm 2.5$ & 0.22 \\
\hline \# of oocytes retrieved & $11 \pm 7.2$ & $6.7 \pm 5.7$ & 0.08 \\
\hline Oocyte recovery rate & $0.85 \pm 0.44$ & $0.97 \pm 0.48$ & 0.29 \\
\hline$\%$ of MII oocytes per oocytes retrieved & $0.92 \pm 0.07$ & $0.78 \pm 0.21$ & 0.09 \\
\hline$\%$ of $2 \mathrm{PN}$ per Mll oocytes retrieved & $0.63 \pm 0.2$ & $0.605 \pm 0.29$ & 0.43 \\
\hline$\%$ of TQE per $2 \mathrm{PN}$ retrieved & $0.6 \pm 0.37$ & $0.59 \pm 0.52$ & 0.48 \\
\hline
\end{tabular}

\section{Discussion}

In the present study of patients undergoing the Stop GnRH-Agonist combined with multiple-dose GnRHantagonist protocol, who underwent triggering of final follicular maturation with $\mathrm{GnRH}$-agonist trigger, either solely or combined with hCG (dual trigger), 5 out of the 32 patients $(15.6 \%)$ demonstrated suboptimal response as reflected by LH levels $<15 \mathrm{IU} / \mathrm{L} 12 \mathrm{hrs}$ post $\mathrm{GnRH}$-agonist trigger. This figure is higher than the reported 5.2\% in patients undergoing the multiple-dose $\mathrm{GnRH}$-antagonist protocol (9). $\mathrm{GnRH}$-agonist causes suppression of pituitary LH secretion for as long as 10 days after the last dose of 
the agonist (15). It might be therefore assumed that a residual prolonged pituitary suppression following the Stop $\mathrm{GnRH}$-agonist protocol might be the culprit of the observed higher suboptimal response.

We could not observe any differences in oocytes recovery rate, maturity or embryos quality between the different study groups. The reason is that the aforementioned variables are affected by the trigger mode, and most of our patients were triggered with both GnRH-agonist and hCG (dual trigger). On the other hand, we could indeed demonstrate significantly higher number of follicles and peak estradiol levels at the day of trigger in those achieving a suboptimal response to the $\mathrm{GnRH}$-agonist trigger (post trigger $\mathrm{LH}<$ $15 \mathrm{mlU} / \mathrm{mL})$.

Therefore, it might be suggested that high responders undergoing the Stop GnRH-agonist combined with $\mathrm{GnRH}$-antagonist are at risk to a suboptimal response to $\mathrm{GnRH}$-agonist solely trigger. To avoid this suboptimal respond, we recommend considering the dual trigger with low-dose hCG (1000-1500IU), in this subgroup of patients, aiming to prevent the suboptimal response.

The Stop GnRH-agonist combined with GnRH-antagonist was successfully used several groups of patients (10-12). The rationale behind the sequential treatment stems from the advantages of its components: (a) The long $\mathrm{GnRH}$-ag protocol pretreatment results in better synchronized response and a scheduled cycle (16-17); (b) Since continuing the $\mathrm{GnRH}$-ag during $\mathrm{COH}$ is often associated with a significant increase in the number of gonadotropin ampoules required for achieving adequate follicular development, its cessation might improve ovarian response and avoids the need of increasing gonadotropin daily dose. GnRH-ag causes suppression of pituitary LH secretion for as long as 10 days after the last dose of the agonist (15); and (c) The Stop GnRH-ag together with the multiple-dose GnRHant provide immediate LH suppression, eliminating premature LH surge and might improve the quality of the embryos generated. In the present study we could demonstrate another advantage, by the feasibility of triggering final oocyte maturation by GnRH agonist together with hCG (Dual trigger), with improved IVF outcome (13).

In conclusion, the Stop $\mathrm{GnRH}$-agonist combined with $\mathrm{GnRH}$-antagonist protocol, has been successfully used in poor responder patients (10), those with poor embryos quality (11) and those with elevated peak serum progesterone levels (12). In the present study we could demonstrate that it also enables the substitution of HCG with GnRH-ag for final oocyte maturation. However, cautioned should be taken in high responders, where the addition of small doses of hCG (1000-1500IU) should be considered (dual trigger), aiming to avoid suboptimal response (post trigger $\mathrm{LH}$ levels $<15 \mathrm{IU} / \mathrm{L}$ ). Further studies are required to identify other risk factors for suboptimal response to $\mathrm{GnRH}$-agonist trigger.

\section{Declarations}

\section{Ethics approval and consent to participate:}

The study protocol was approved by the "Sheba Medical Center" Institutional Review Board. 
Availability of data and materials:

Data will be made available from the corresponding author on request.

\section{Competing interests:}

The authors have nothing to declare.

\section{Funding:}

This manuscript was not supported by specific funding.

\section{Consent for publication:}

Not applicable (cohort historical)

\section{Authors' contributions:}

RO- wrote the paper and edited it in all its revisions., performed the statistical evaluations took part in discussions regarding the results.

RN-Participated in designing the study, retrieved the data proof read the paper and took part in discussions regarding the results.

JF- Retrieved the data, proof read the paper and took part in discussions regarding the results.

YF- Retrieved the data, proof read the paper and took part in discussions regarding the results.

OZ- Retrieved the data, performed laboratory work, proof read the paper and took part in discussions regarding the results.

$\mathrm{JH}$ - Participated in designing the study, assisted in writing the paper and edited it, proof read the paper and took part in discussions regarding the results.

\section{Acknowledgment:}

NA

\section{References}

1. Penzias AS. Improving results with assisted reproductive technologies: individualized patient-tailored strategies for ovulation induction. Reprod Biomed Online 2004;9:43-6.

2. Mersereau J, Stanhiser J, Coddington C, Jones T, Luke B, Brown MB. Patient and cycle characteristics predicting high pregnancy rates with single-embryo transfer: an analysis of the Society for Assisted Reproductive Technology outcomes between 2004 and 2013. Fertil Steril 2017;108(5):750-756. 
3. The Practice Committee of the American Society for Reproductive Medicine (ASRM). Prevention and treatment of moderate and severe ovarian hyperstimulation syndrome: a guideline. Fertil Steril 2016;106(7):1634-1647

4. Kol S. Luteolysis induced by a gonadotropin-releasing hormone agonist is the key to prevention of ovarian hyperstimulation syndrome. Fertil Steril 2004;81:1-5

5. Orvieto R. Ovarian hyperstimulation syndrome- an optimal solution for an unresolved enigma. J Ovarian Res 2013;6(1):77.

6. Kummer NE, Feinn RS, Griffin DW, Nulsen JC, Benadiva CA, Engmann LL. Predicting successful induction of oocyte maturation after gonadotropin-releasing hormone agonist ( $\mathrm{GnRHa}$ ) trigger. Hum Reprod 2013;28:152-9.

7. Chen SL, Ye DS, Chen X, Yang XH, Zheng HY, Tang Y, et al. Circulating luteinizing hormone level after triggering oocyte maturation with $\mathrm{GnRH}$ agonist may predict oocyte yield in flexible $\mathrm{GnRH}$ antagonist protocol. Hum Reprod 2012;27:1351-6.

8. Shapiro BS, Daneshmand ST, Restrepo H, Garner FC, Aguirre M, Hudson C. Efficacy of induced luteinizing hormone surge after "trigger" with gonadotropin-releasing hormone agonist. Fertil Steril 2011;95:826-8.

9. Meyer L, Murphy LA, Gumer A, Reichman DE, Rosenwaks Z, Cholst Risk factors for a suboptimal response to gonadotropin-releasing hormone agonist trigger during in vitro fertilization cycles. Fertil Steril 2015;104:637-642.

10. Orvieto R, Kirshenbaum M, Galiano V, Elkan-Miller T, Zilberberg E, Haas J, Nahum R. Stop GnRHAgonist Combined With Multiple-Dose GnRH-Antagonist Protocol for Patients With "Genuine" Poor Response Undergoing Controlled Ovarian Hyperstimulation for IVF. Front Endocrinol (Lausanne). 2020;11:182.

11. Orvieto R, Kirshenbaum M, Galiano V, Zilberberg E, Haas J, Nahum R. Stop GnRH-agonist combined with multiple-dose $\mathrm{GnRH}$-antagonist for patients with elevated peak serum progesterone levels undergoing ovarian stimulation for IVF: A proof of concept. Gynecol Obstet Invest 85: 357-361.

12. Orvieto R, Kirshenbaum M, Galiano V, Zilberberg E, Haas J, Nahum R. Stop GnRH-Agonist Combined with Multiple-Dose GnRH-Antagonist for Patients with Elevated Peak Serum Progesterone Levels Undergoing Ovarian Stimulation for IVF: A Proof of Concept. Gynecol Obstet Invest 2020;85(4):357361.

13. Orvieto R. Triggering final follicular maturation-hCG, GnRH-agonist or both, when and to whom? J Ovarian Res. 2015;8:60.

14. Ziebe S, Lundin K, Janssens R, Helmgaard L, Arce JC, Group M. Influence of ovarian stimulation with HP-hMG or recombinant FSH on embryo quality parameters in patients undergoing IVF. Hum Reprod 2007;22:2404-13.

15. Pritts EA, Atwood AK. Luteal phase support in infertility treatment: a meta-analysis of the randomized trials. Hum Reprod 2002;17:2287-99. 
16. Huirne JA, Homburg R, Lambalk CB.Are GnRH antagonists comparable to agonists for use in IVF? Hum Reprod. 2007;22(11):2805-13.

17. Orvieto R, Patrizio P. GnRH agonist versus $\mathrm{GnRH}$ antagonist in ovarian stimulation: an ongoing debate. Reprod Biomed Online. 2013;26(1):4-8. 